\title{
Direct heart shunt placement for CSF diversion: technical note
}

\author{
Ziev B. Moses, MD, ${ }^{1}$ Alp Ozpinar, MD, ${ }^{2}$ Muhammad M. Abd-El-Barr, MD, PhD, ${ }^{1}$ \\ Luis G. Quinonez, MD, ${ }^{3}$ Sitaram M. Emani, MD, ${ }^{3}$ and Liliana C. Goumnerova, MD1 \\ Departments of ${ }^{1}$ Neurosurgery and ${ }^{3}$ Cardiac Surgery, Boston Children's Hospital, Harvard Medical School, Boston, \\ Massachusetts; and ${ }^{2}$ Oregon Health and Science University, Portland, Oregon
}

\begin{abstract}
The authors report a complex case of an 18-year-old male with a history of hydrocephalus secondary to intraventricular hemorrhage of prematurity, with more than 30 previous shunt revisions, who presented to the authors' institution with shunt malfunction. After exhausting his peritoneal cavity and pleural space as possible distal sites of shunt placement, he underwent a direct heart shunt placement when it was discovered he had thrombosis of his subclavian vein precluding a standard wire-guided atrial cannulation. His course was complicated by postoperative distal catheter migration and repeat surgery for reimplantation of the shunt directly into the atrium. At the 16-month follow-up visit, the patient showed no symptoms of shunt malfunction or pericardial effusion. Imaging studies demonstrated a functioning shunt system. This is the second reported successful ventricle to direct heart shunt placement in an adult. The authors report on the technical aspects of the case and review the relevant literature.
\end{abstract}

http://thejns.org/doi/abs/10.3171/2016.5.PEDS15638

KEY WORDS hydrocephalus; shunt; cardiac; technique

$\mathrm{D}$ IRECT heart shunts were more commonly used as a method of CSF diversion for hydrocephalus in the 1950 s as an alternative self-lengthening shunt system when many surgeons continued to be hesitant toward diverting CSF to the peritoneum. ${ }^{4}$ Technical and material developments have since made ventriculoperitoneal (VP) shunts more reliable and associated with fewer long-term complications, specifically septicemia with secondary CSF infection and renal insufficiency. However, certain patients require alternative CSF diversion approaches due to their medical history and/or surgical anatomy. Distal placement of CSF shunts into atria, pleural space, the gallbladder, bone marrow, and directly into the heart itself have all been described. ${ }^{2,3,6,8,10,13}$ In this paper we present the case of an 18-year-old male who, after having undergone multiple shunt revisions, ultimately required placement of a direct ventricle to heart shunt.

\section{Case Presentation}

\section{History and Examination}

This 18-year-old male, who had a shunt placed shortly after birth due to hydrocephalus secondary to intra- ventricular hemorrhage of prematurity, presented with a 6-week onset of headaches, difficulty sleeping, and lowgrade fevers. He had a history of multiple shunt revisions, including multiple distal revisions at the peritoneum and 2 pleural site revisions. At presentation, he had a ventriculoatrial (VA) shunt that was explored, and intraoperative attempts to thread a wire into the atrium failed, suggesting a possible clot. The VA shunt was removed and a VP shunt was placed. Postoperatively, a diagnostic venogram demonstrated partial occlusion of the right subclavian vein, near complete occlusion of the right internal jugular vein, and a high-grade stenosis at the junction of the right brachiocephalic vein and superior vena cava. Two days after placement of the VP shunt he developed a fever and CSF cultures grew Propionibacterium acnes. The shunt was externalized and he was started on vancomycin. Peritoneal cultures grew gram-negative rods and he was treated for Serratia peritonitis. Nine days later he was taken back to the operating room for internalization of his shunt into the pleural cavity. Postoperatively he did well for 1 day but he continued to have fevers and increasing respiratory distress, and imaging demonstrated a large pleural fluid collection. He was subsequently taken back to the operating

ABBREVIATIONS EVD = external ventricular drain; ICU = intensive care unit; VA = ventriculoatrial; VATS = video-assisted thoracoscopic surgery; VP = ventriculoperito-

neal.

SUBMITTED November 1, 2015. ACCEPTED May 25, 2016.

INCLUDE WHEN CITING Published online September 2, 2016; DOI: 10.3171/2016.5.PEDS15638. 


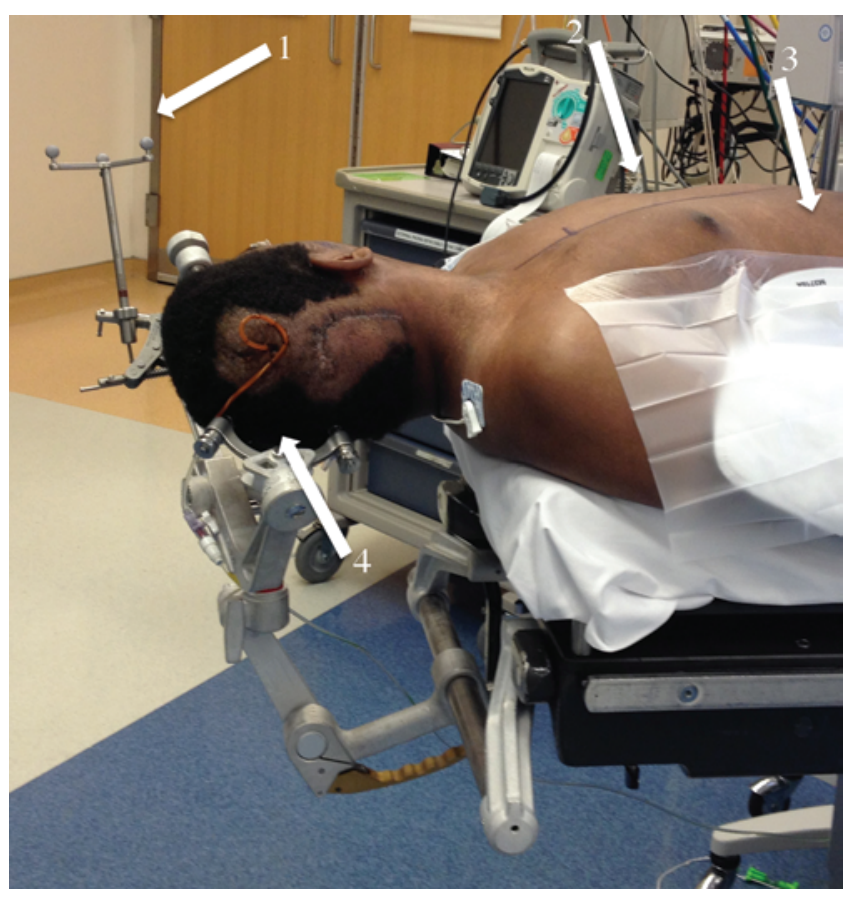

FIG. 1. Photograph of the intraoperative positioning: 1) image guidance reference array; 2) midline pericardial incision marking; 3 ) defibrillator pad; and 4) EVD catheter and cranial shunt incision. Figure is available in color online only.

room for externalization of the right-sided pleural catheter and fluid drainage via video-assisted thoracoscopic surgery (VATS). A new temporary external ventricular drain (EVD) was placed.

Given the above complications and need for CSF diversion, a decision to place a direct VA shunt was made. Cardiothoracic surgery was consulted for direct placement of the distal catheter into the right atrium via median sternotomy.

\section{Technical Details}

The patient was brought to the operating room and the surgery was performed in a designated cardiac surgery room. Operative positioning was performed such that we could simultaneously access the cranial sites as well as the chest for placement of the shunt. Given his complex ventricular anatomy, his head was immobilized in the Mayfield headrest and he was registered into the BrainLab system for placement of the ventricular catheter into the right lateral ventricle (Fig. 1). Both cardiac surgery and neurosurgery teams were present and the draping included all incisions.

Prior to incision, the patient had defibrillator paddles placed should intraoperative cardioversion be required. Intraoperative navigation was used for placement of the proximal catheter. After placement of the proximal ventricular catheter, a midline epigastric incision was used to create a pericardial window (Fig. 2). Two purse-string sutures were placed on the free wall of the right atrium. The distal components of the shunt consisting of a medium fixed-pressure valve and distal peritoneal tubing were then

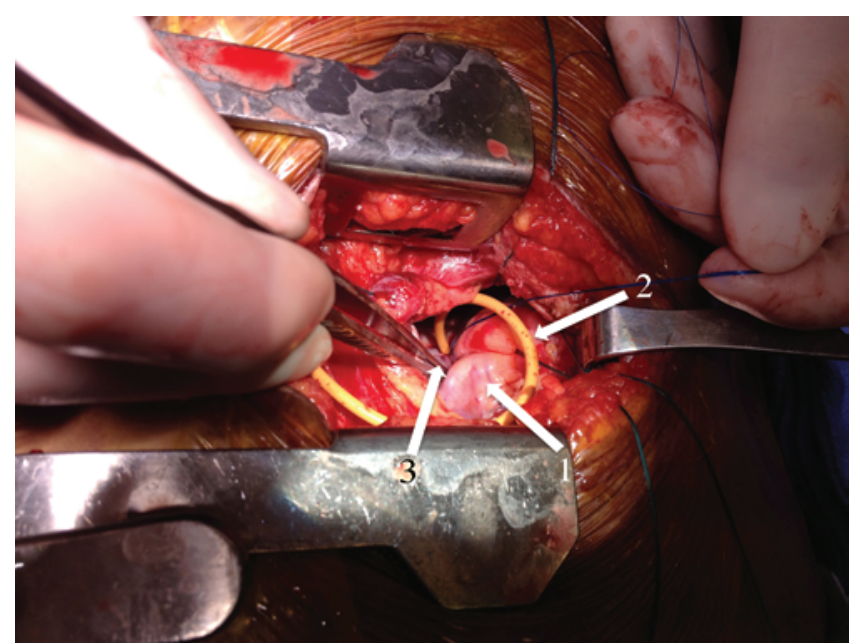

FIG. 2. Photograph of the pericardial window: 1) anterior free wall of right ventricle; 2) traction suture on atrioventricular groove; and 3) pursestring suture at the entry site of the Bactiseal catheter into the right atrium. Figure is available in color online only.

implanted into the patient. Two-step incisions were used to tunnel the catheter to the epigastric incision from the cranial incision. The shunt tubing was placed through the abdominal wall fascia and musculature into the pericardial window. A stab incision was then made in the body of the right atrium and the tip was then advanced inferiorly into the atrium for a distance of $2 \mathrm{~cm}$. The 2 purse-string sutures were tied, securing hemostasis. A 19 Fr Blake drain (Ethicon) was placed in the pericardium. Standard closure was performed for all incisions and the patient was then awoken from anesthesia and transported to the intensive care unit (ICU), primarily to monitor for cardiac arrhythmias. A postoperative CT scan confirmed the location of the proximal catheter tip in the right lateral ventricle.

\section{Follow-Up}

The patient was monitored in the ICU, and 2 days following placement chest tube output was found to increase in quantity. A chest radiograph revealed that the distal catheter had migrated compared with the immediate postoperative film (Fig. 3). A follow-up CT scan showed that the end of the tubing was in the pericardial space (Fig. 4). The patient was taken back to the OR for distal catheter revision. The epigastric incision was opened. The tip of the shunt was found in the pericardial space. The previous purse-string suture was intact. The surface of the shunt was felt to be slippery as it was antibiotic coated, and migration was attributed to this surface characteristic. A new purse-string suture was placed in the atrium, through which the catheter was reintroduced into the atrium. To prevent recurrent migration, a silk suture was tied directly to the catheter $2 \mathrm{~cm}$ from the tip, with care taken to avoid compression of the catheter lumen. The silk suture was used to secure the catheter to the purse-string suture to prevent migration. A chest tube was replaced, and the incision was then closed in layers. The patient was transferred back to the ICU in stable condition. A postoperative chest radiograph revealed a stable catheter tip in a location 


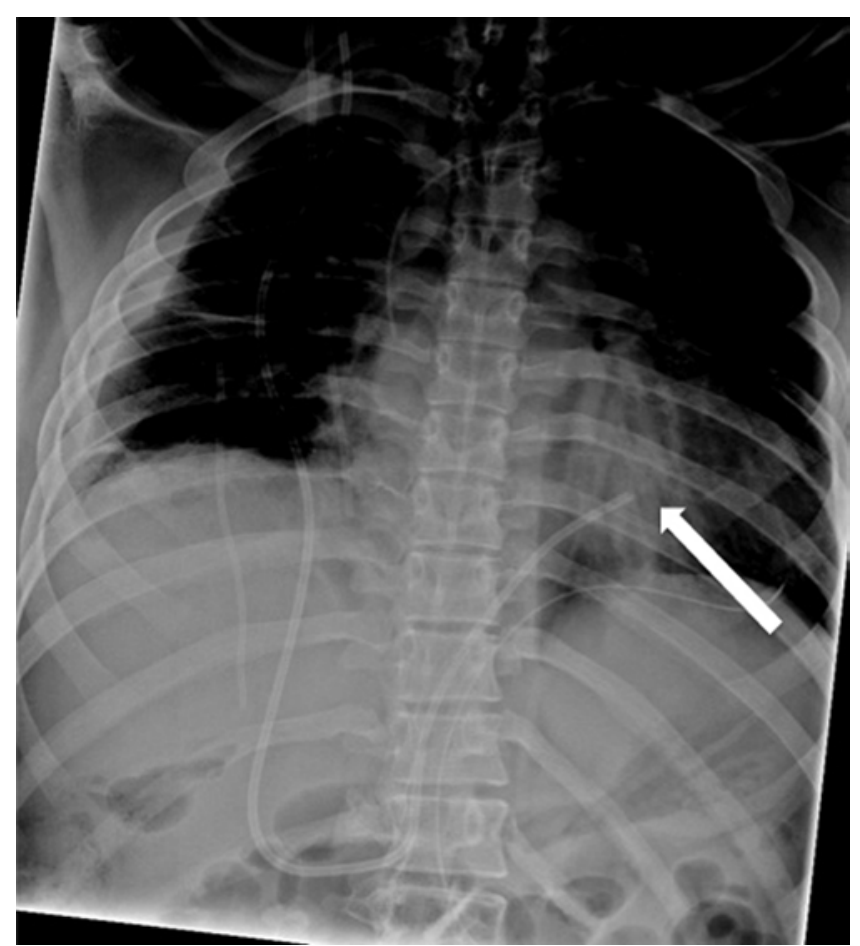

FIG. 3. Pre-revision chest radiograph revealing the catheter tip (arrow) migrated from the right atrium inferiorly to the right pericardial space.

compatible with the right atrium over the course of the patient's hospital stay (Fig. 5). Working together with the infectious disease team, an antibiotic regimen was formulated for the patient that included of a total of 2 weeks of vancomycin for the $P$. acnes infection while the patient was externalized and an additional 4 weeks of cefepime for the Serratia infection following his initial VATS procedure.

At the 16-month follow-up the patient remained asymptomatic and imaging studies demonstrated a functioning shunt system in the correct location.

\section{Discussion}

CSF shunt placement is one of the most commonly performed procedures in the neurosurgical profession. ${ }^{1} \mathrm{VP}$ shunts are the mainstay for the treatment of hydrocephalus and have demonstrated good long-term outcomes. Compared with other CSF diversion techniques, VP shunts require fewer revisions to accommodate patient growth and are associated with fewer morbid infections. ${ }^{4,11}$ Regardless of the etiology necessitating VP shunt placement, these devices eventually fail over time. Additionally, preexisting conditions can preclude use of the peritoneal cavity as a CSF reservoir, requiring additional sites to be identified. When this is the case, the pleural space, gall bladder, urinary bladder, atrium via venous vasculature, and atrium via direct cardiac insertion are acceptable choices for CSF diversion sites. . $^{3,8,10,13}$

Prior to the advent of Silastic VP shunt tubing, placement of non-Silastic tubing into the peritoneal cavity was cumbersome and often associated with significant perito-

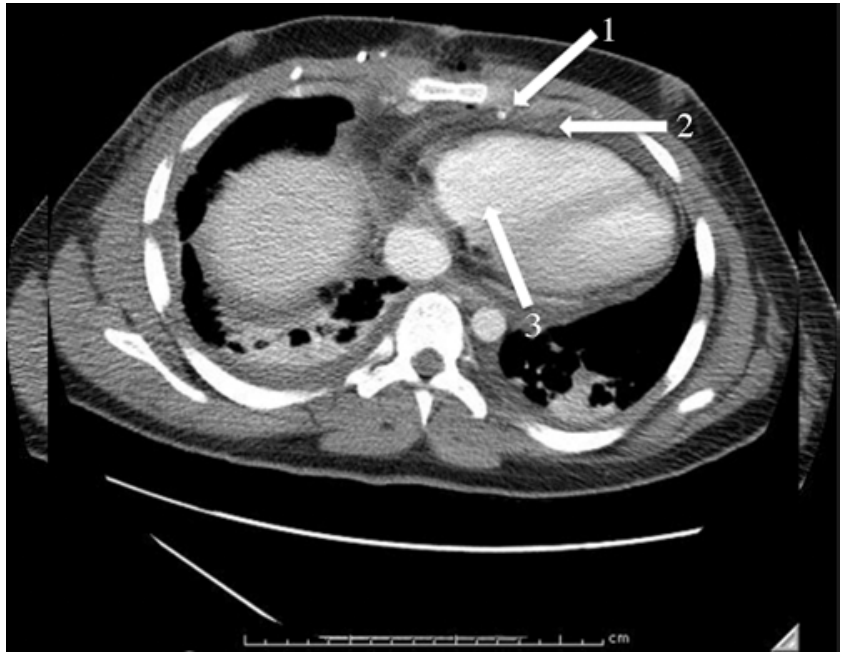

FIG. 4. CT scan of the chest confirming the catheter tip (1) in the pericardial space (2) with effusion. 3 = right ventricle.

neal irritation. ${ }^{7}$ For this reason, in the period between the late 1960s and the early 1970s, direct heart shunts were frequently preferred to transvenous VA shunts. In a case series published in 1967 that involved 42 pediatric patients, Weinman and Paul found that some of the advantages of direct heart shunts over transvenous VA shunts were: 1) accurate placement of the shunt; 2) ability to allow for growth; 3) lack of interference with venous return; and 4) avoidance of jugular or caval thrombosis. ${ }^{12}$ Reported complications in their study included 2 deaths from postoperative pulmonary infections and 1 case of pneumothorax. ${ }^{12}$

More recently, Martin et al. evaluated the durability and efficacy of direct heart shunts in 60 pediatric patients. The mean shunt life in their series was 79 months with a distal shunt revision rate of $47.1 \% .^{5}$ Complication rates were similar to those reported by Weinman and Paul, and

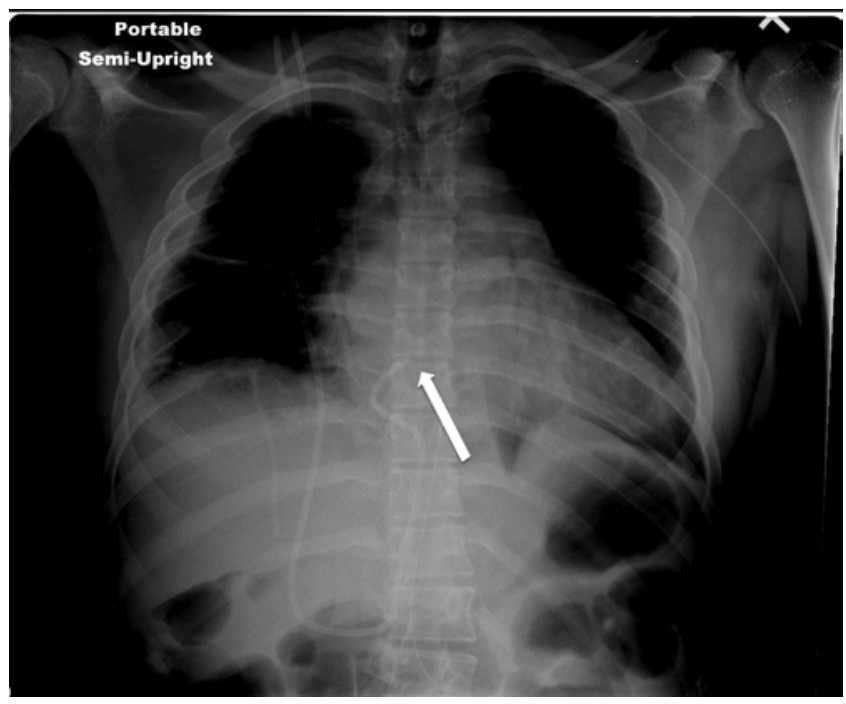

FIG. 5. Post-revision chest radiograph with the catheter tip (arrow) in the area corresponding to the right atrium. 
consisted of 1 case of pneumothorax and 2 deaths due to infection. These results are favorable to the earlier case series of venous VA shunts that demonstrated a mean shunt life of 62.6 months and a distal revision rate of $79.9 \% .^{12}$

Our technique differs from that of Martin et al. in that we use a subxiphoid rather than inframammary incision, the catheter is brought out in a subcostal rather than intercostal location, and we insert the catheter in the body of the atrium instead of the atrial appendage., ${ }^{5,12}$

Finally, an important technical consideration is the method of securing the antibiotic-coated catheter to the atrium. With time, the antibiotic coating of the catheter becomes progressively more hydrated, which predisposes it to slipping within the purse-string. Additional methods of fixation to the surface of the atrium include using a commercially available catheter stabilizer or traction suture.

In this paper we report a successful placement of a direct heart shunt in an 18-year-old male in whom all other alternatives had failed. This is the second reported case of a successfully placed shunt using the direct ventricle to heart technique in an adult (Medline search criteria keywords: "CSF diversion," "direct heart shunt," and "direct cardiac shunt").

\section{Conclusions}

Today, direct heart shunts are a seldom-used CSF diversion technique used in cases where other distal sites are preluded by preexisting conditions and there is a fear of jugular and caval thrombosis. However, this technique remains effective with revision rates and complications favorable to transvenous VA shunts. It should be carefully considered as a salvage CSF diversion technique. This is the second reported case of a successfully placed shunt using the direct ventricle to heart technique in an adult. ${ }^{9}$

\section{References}

1. Bondurant CP, Jimenez DF: Epidemiology of cerebrospinal fluid shunting. Pediatr Neurosurg 23:254-259, 1995

2. Hammock MK, Milhorat TH, McClenathan JE: Expanding ventricular shunts for hydrocephalus in infancy and childhood. Dev Med Child Neurol Suppl 35:89-93, 1975

3. Kempe LG, Blaylock R: Ventriculolymphatic shunt. J Neurosurg 47:86-95, 1977

4. Keucher TR, Mealey J Jr: Long-term results after ventriculoatrial and ventriculoperitoneal shunting for infantile hydrocephalus. J Neurosurg 50:179-186, 1979

5. Martin JE, Keating RF, Cogen PH, Midgley FM: Long-term follow-up of direct heart shunts in the management of hydrocephalus. Pediatr Neurosurg 38:94-97, 2003

6. Milhorat TH, McClenathan JE: Direct cardiac shunt for hydrocephalus of infancy and childhood. Technical note. J Neurosurg 42:605-608, 1975

7. Scarff JE: Treatment of hydrocephalus: an historical and critical review of methods and results. J Neurol Neurosurg Psychiatry 26:1-26, 1963

8. Stringel G, Turner M, Crase T: Ventriculo-gallbladder shunts in children. Childs Nerv Syst 9:331-333, 1993

9. Thompson EM, Giles SG, Song HK, Litvack ZN, Golshani KJ, Nemecek AN: Successful ventricle to direct heart shunt placement as a salvage cerebrospinal fluid diversion technique. Case report. J Neurosurg Pediatr 3:521-524, 2009

10. Venes JL, Shaw RK: Ventriculopleural shunting in the management of hydrocephalus. Childs Brain 5:45-50, 1979

11. Vernet $\mathrm{O}$, Campiche R, de Tribolet $\mathrm{N}$ : Long-term results after ventriculoatrial shunting in children. Childs Nerv Syst 9:253-255, 1993

12. Weinman D, Paul AT: Ventriculoauriculostomy for infantile hydrocephalus using a direct cardiac approach. Technical note. J Neurosurg 27:471-474, 1967

13. Weiss MH, Jane JA, Apuzzo ML, Heiden JS, Kurze T: Ventriculogastrostomy, an alternative means for CSF diversion: a preliminary study. Bull Los Angeles Neurol Soc 40:140144,1975

\section{Disclosures}

The authors report no conflict of interest concerning the materials or methods used in this study or the findings specified in this paper.

\section{Author Contributions}

Conception and design: Goumnerova. Acquisition of data: Goumnerova, Moses, Ozpinar, Quinonez, Emani. Analysis and interpretation of data: Goumnerova, Moses, Ozpinar. Drafting the article: Goumnerova, Moses, Ozpinar, Abd-El-Barr. Critically revising the article: all authors. Reviewed submitted version of manuscript: Goumnerova, Moses. Study supervision: Goumnerova.

\section{Supplemental Information \\ Current Affiliations}

Dr. Ozpinar: Department of Neurological Surgery, University of Pittsburgh, Pittsburgh, PA.

\section{Correspondence}

Liliana C. Goumnerova, Department of Neurosurgery, Hunnewell-2, Boston Children's Hospital, 300 Longwood Ave., Boston, MA 02115.email: liliana.goumnerova@childrens. harvard.edu. 